\title{
Development Status of Type II Superlattice Infrared Detector in JAXA
}

\author{
Haruyoshi Katayama*, Michito Sakai, Junpei Murooka, \\ Masafumi Kimata ${ }^{1}$, Takahiro Kitada ${ }^{2}$, Toshiro Isu ${ }^{2}$, \\ Mikhail Patrashin ${ }^{3}$, Iwao Hosako ${ }^{3}$ and Yasuhiro Iguchi ${ }^{4}$ \\ Earth Observation Research Center, Japan Aerospace Exploration Agency, \\ 2-1-1 Sengen, Tsukuba, Ibaraki 305-0035, Japan \\ ${ }^{1}$ College of Science and Engineering, Ritsumeikan University, \\ 1-1-1 Noji-higashi, Kusatsu, Shiga 525-8577, Japan \\ ${ }^{2}$ Institute of Technology and Science, The University of Tokushima, \\ 2-1 Minamijyousanjima-cho, Tokushima 770-8506, Japan \\ ${ }^{3}$ Advanced ICT Research Institute, National Institute of Information and \\ Communications Technology, 4-2-1 Nukui-Kitamachi Koganei, Tokyo 184-8795, Japan \\ ${ }^{4}$ Transmission Devices R\&D Laboratories, Sumitomo Electric Industries, Ltd., \\ 1 Taya-cho, Sakae-ku, Yokohama 244-8588, Japan
}

(Received December 11, 2013; accepted March 24, 2014)

Key words: InAs/GaSb Type II superlattice infrared detectors, mid-wave infrared

We present the development status of a Type II superlattice (T2SL) infrared detector for future space applications in JAXA. InAs/GaSb T2SL is the only known infrared material that has a theoretically predicted higher performance than $\mathrm{HgCdTe}$. We first fabricated a single-pixel detector with a cutoff wavelength of $6 \mu \mathrm{m}$. The detector is a pin photodiode with a superlattice consisting of 9 InAs monolayers (MLs) and $7 \mathrm{GaSb}$ MLs. The dark current density of the detector is $1.8 \times 10^{-4} \mathrm{~A} / \mathrm{cm}^{2}$ at a bias voltage of $-100 \mathrm{mV}$. We also present the results of an optical evaluation of the detector. The cutoff wavelength is $5.5 \mu \mathrm{m}$ at $30 \mathrm{~K}$. The responsivity is $0.3 \pm 0.05 \mathrm{~A} / \mathrm{W}$ at $4.5 \mu \mathrm{m}$. We also show the development of an array detector with a cutoff wavelength of $6 \mu \mathrm{m}$. However, further improvements are required for developing array detectors with longer cutoff wavelengths.

\section{Introduction}

Infrared sensors are key for realizing many missions that are part of the future mission roadmap for Japan Aerospace Exploration Agency (JAXA), such as Earth observations, meteorological observations, disaster monitoring, and planetary explorations. Infrared detectors are the most important component of infrared sensors for realizing these

*Corresponding author: e-mail: katayama.haruyoshi@jaxa.jp 
missions. To meet more demanding mission requirements, JAXA has decided to position infrared detector technology as a strategic technology and promote its development.

The "Geostationary Meteorology and Atmospheric Chemistry Mission" is one of the future missions of JAXA. ${ }^{(1)}$ Conventional observation satellites positioned at the low Earth orbit do not pass over the same location at a sufficient frequency; therefore, such satellites are not effective for tracing the photochemical processes of pollutants and their intercontinental transfers, which are suspected to be behind the recent increase in air pollution in Japan. A geostationary satellite is the best platform to monitor such phenomena. In addition, there is strong demand for future geostationary meteorology to improve numerical weather prediction by frequent sounding of the temperature, water vapor, and wind profiles.

It is planned that the satellite will consist of two optical sensors: ultraviolet/visible and infrared. The baseline of the infrared sensor system is an imaging Fourier transform spectrometer (FTS). The imaging FTS simultaneously acquires images and spectra by using an array detector on its focal plane. The focal plane array (FPA) of the imaging FTS should have high sensitivity and a very long wave infrared (VLWIR) cutoff wavelength of $15 \mu \mathrm{m}$.

The Type II superlattice (T2SL) is the only known infrared material to exhibit performance that is theoretically predicted to be higher than that of $\mathrm{HgCdTe}$. T2SL can avoid nonuniformity of FPA because of fluctuations in the alloy composition, particularly in the VLWIR region. (2) Our final goal for developing the T2SL detector is to realize a T2SL detector array with a cutoff wavelength $\lambda_{\mathrm{c}}$ of $15 \mu \mathrm{m}$. In this paper, we present the T2SL detector development in JAXA.

\section{InAs/GaSb Type II Strained Layer Superlattice}

The T2SL detector was proposed by Smith and Mailhiot ${ }^{(3)}$ in 1987. The detector uses characteristics of the bandgap between InAs and $\mathrm{Ga}(\mathrm{In}) \mathrm{Sb}$. As shown in Fig. 1, InAs and GaSb show that a misaligned Type II band alignment with the conduction band of the InAs is lower than the valence band of the GaSb. The effective bandgap, i.e., the cutoff wavelength, can be tailored in the wavelength region of 3-30 $\mu \mathrm{m}$ by adjusting the thickness of the individual layers and the indium content.

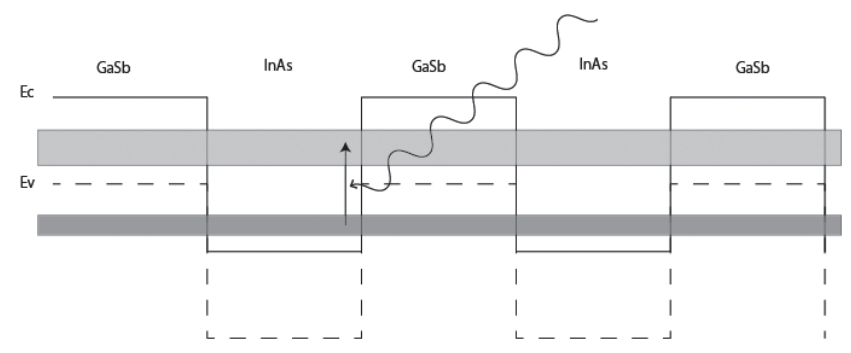

Fig. 1. Schematic band diagram of GaSb/InAs Type II superlattice. 
The T2SL detector is predicted to yield a lower dark current, higher operating temperature than traditional cooled infrared detectors, and improved spatial uniformity. Thus, the T2SL detector is said to have the potential to replace $\mathrm{HgCdTe}$ for longwavelength infrared detector applications. The performance of the T2SL detector has been improved over the years, ${ }^{(4,5)}$ and large-format focal plane arrays ${ }^{(6)}$ and dual-color focal plane arrays ${ }^{(7)}$ have been realized.

\section{Growth of T2SL Crystal}

The performance of the T2SL detector strongly depends on the crystal quality. Thus, we focused on crystal growth as the first step in the development of the T2SL detector. Figure 2 (left) shows the design of the T2SL crystal sample that we fabricated. We began from the mid-wave infrared (MWIR) T2SL detector with a cutoff wavelength of $6 \mu \mathrm{m}$ because it is still difficult to suppress the dark current for the longer wavelength detector. The T2SL crystal sample was designed for pin diodes with a superlattice having a thickness of 9 monolayers (MLs) of InAs and 7 MLs of GaSb. To compensate for the residual tensile strain due to the lattice mismatch between InAs and GaSb, 0.9 MLs of $\mathrm{InSb}$ were grown between the InAs and GaSb layers.

The samples were grown by solid-source molecular beam epitaxy (MBE) using a Veeco Gen II system at the National Institute of Information and Communications Technology, Japan. First, a 0.5 - $\mu \mathrm{m}$-thick GaSb buffer layer doped with $\mathrm{Be}\left(p \approx 2 \times 10^{18}\right.$ $\left.\mathrm{cm}^{-3}\right)$ was deposited. A 50-period InAs/GaSb:Be $\left(p \approx 1 \times 10^{17} \mathrm{~cm}^{-3}\right)$ superlattice was then grown, which was followed by the growth of a 20-period undoped superlattice. Finally, a 30-period InAs: $\mathrm{Si} / \mathrm{GaSb}\left(n \approx 1 \times 10^{17} \mathrm{~cm}^{-3}\right)$ superlattice was grown and capped with 20-nm-thick InAs:Si $\left(n \approx 2 \times 10^{18} \mathrm{~cm}^{-3}\right)$ as a top contact layer. The growth rate was $0.5 \mathrm{MLs} / \mathrm{s}$ for the InAs and GaSb layers. Figure 2 (right) shows the cross section of the T2SL crystal, as obtained by transmission electron microscopy (TEM).

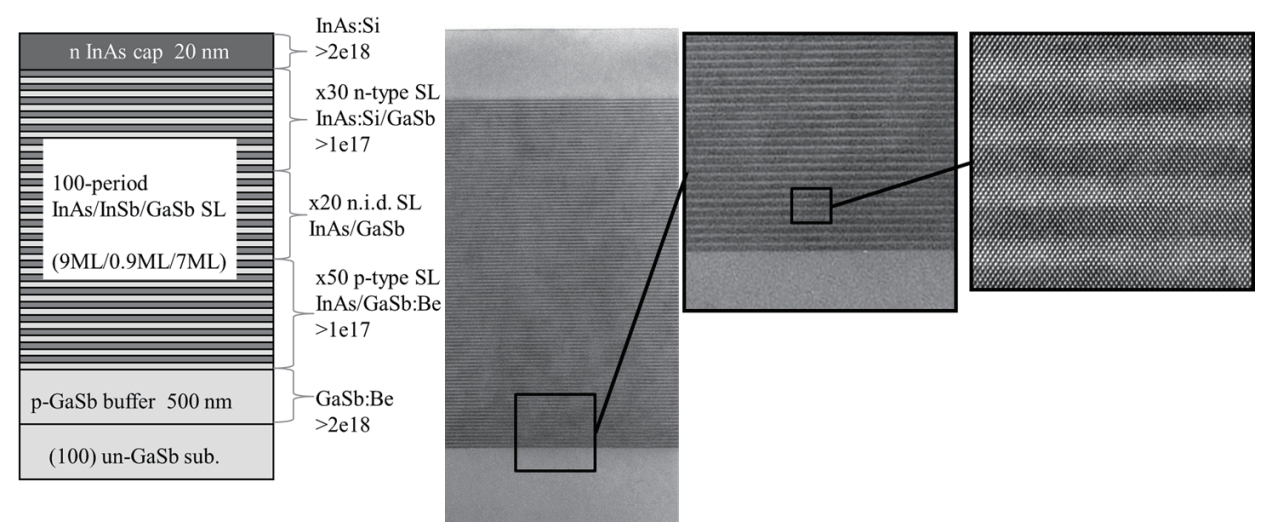

Fig. 2. Design (left) and transmission electron microscopy images (right) of T2SL crystal. 
Figure 3 shows the X-ray diffraction (XRD) scan of the T2SL crystal. The XRD spectrum shows a GaSb substrate peak very close to the zero-order superlattice peak and several satellite peaks due to the superlattice, which suggests a high-quality material. The mismatch between the substrate and superlattice layers was calculated from the angular difference between the substrate and superlattice peaks. We measured a mismatch of $\Delta a / a=-0.006 \%$, where $a$ is the lattice parameter of the GaSb substrate and $\Delta a$ is the difference between the substrate and superlattice layers.

\section{Fabrication of T2SL Single-Pixel Detector}

After the growth of the T2SL crystal, the T2SL crystal sample was processed into a single-pixel detector. Figure 4 shows the schematic structure of the T2SL singlepixel detector. Quadratic mesa diodes with sizes varying between $100 \times 100$ and 900 $\times 900 \mu \mathrm{m}^{2}$ were processed as the test element group. The detector is defined by a mesa structure with wet etching. We used a chemical solution based on $\mathrm{H}_{3} \mathrm{PO}_{4} / \mathrm{H}_{2} \mathrm{O}_{2} / \mathrm{H}_{2} \mathrm{O} /$ $\mathrm{C}_{6} \mathrm{H}_{8} \mathrm{O}_{7}$ at room temperature. ${ }^{(8)}$ After wet etching, the sample was immersed in sodium hypochlorite $(\mathrm{NaClO})$ diluted in deionized water. Ohmic contact metallization was performed with $\mathrm{Ti} / \mathrm{Pt} / \mathrm{Au}=500 \AA / 500 \AA / 3000 \AA$ as the top and bottom contacts. SiN was used for passivation. Figure 5 shows a scanning electron microscopy (SEM) image of the T2SL single-pixel detector.

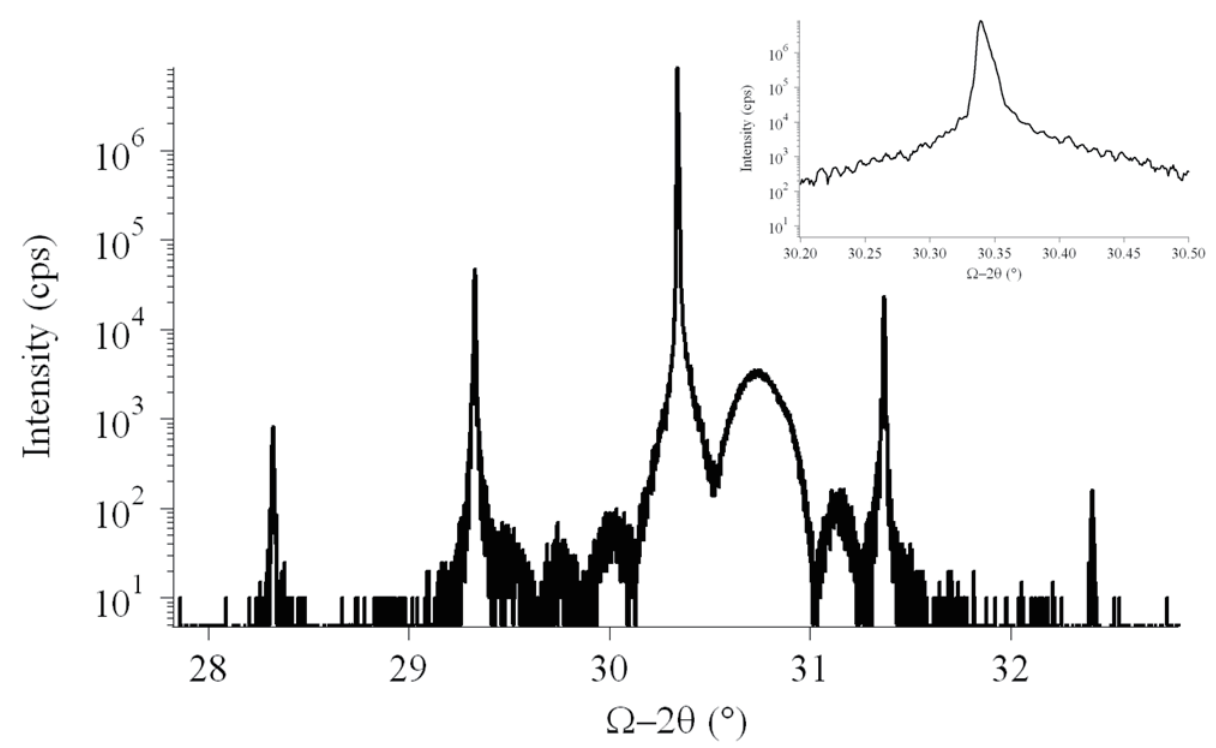

Fig. 3. X-ray diffraction scan of T2SL crystal. Right panel shows a close-up view of the substrate and zero-order satellite peak. 

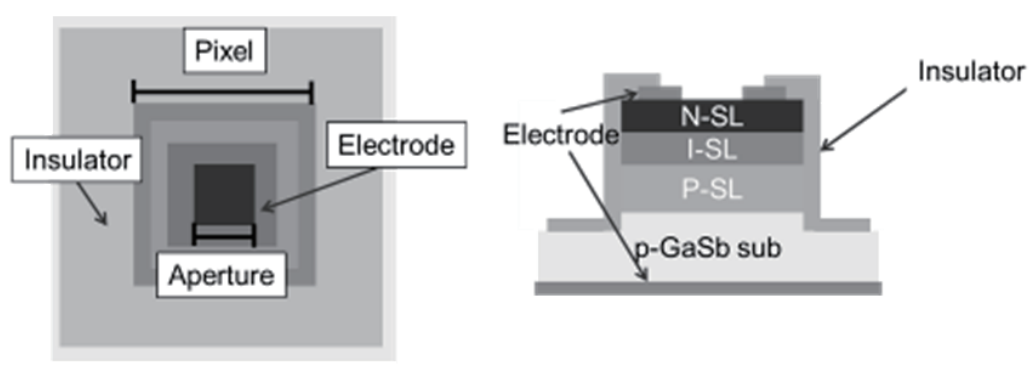

Fig. 4. Schematic structure of T2SL single-pixel detector.

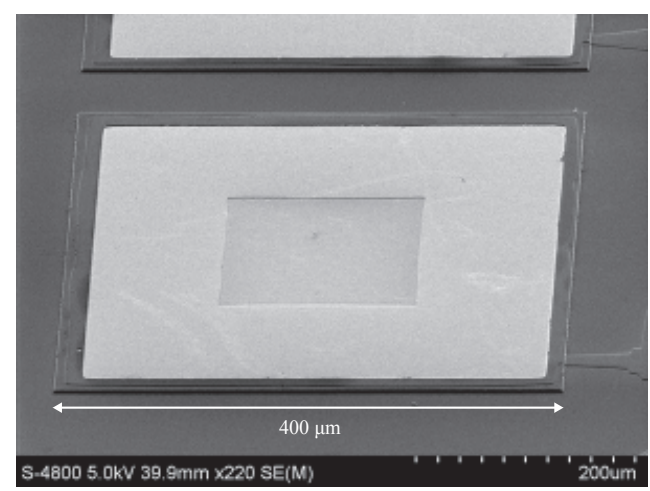

Fig. 5. SEM image of T2SL single-pixel detector. Mesa size is $400 \times 400 \mu \mathrm{m}^{2}$.

\section{Performance Evaluation of T2SL Detector}

The T2SL detector described in $\S 4$ was wire-bonded to a leadless chip carrier package and attached to the cold finger of a cryostat with a Ge window. The cryostat can cool the detector to $30 \mathrm{~K}$ with a He cryocooler (Iwatani D105 and SA112). Using this system, we evaluated the dark current and photometric response of the T2SL detector. In this section, we describe the performance evaluation results of the T2SL detector.

\subsection{Dark current measurement}

The dark current was measured in the temperature range of $30-300 \mathrm{~K}$ with a semiconductor device analyzer (Agilent B1500A). Figure 6 shows the dark current density as a function of the applied bias voltage at $77 \mathrm{~K}$. The dark current density was $1.8 \times 10^{-4} \mathrm{~A} / \mathrm{cm}^{2}$ at a bias voltage of $-100 \mathrm{mV}$. Reference 9 showed that the dark current density is $2 \times 10^{-8} \mathrm{~A} / \mathrm{cm}^{2}$ at $77 \mathrm{~K}$, which is four orders of magnitude lower than that of our detector. 


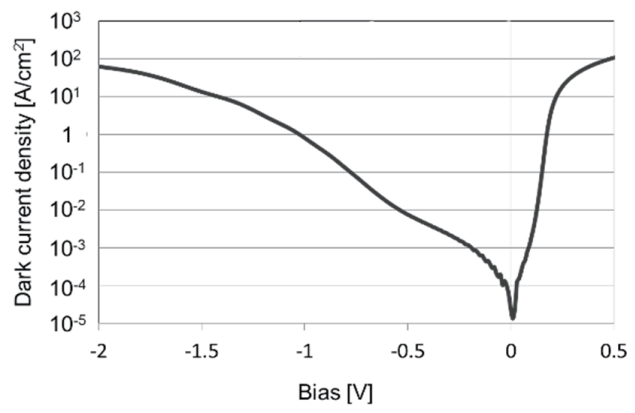

Fig. 6. Dark current density as a function of applied bias voltage at $77 \mathrm{~K}$.

Figure 7 shows an Arrhenius plot of the dark current density at a bias voltage of $V_{\mathrm{b}}$ $=-20 \mathrm{mV}$. The activation energy measured from the Arrhenius plot of the dark current density was $0.22 \mathrm{eV}$, which was very close to the design value of the optical bandgap $(0.21$ $\mathrm{eV})$ for a $6 \mu \mathrm{m}$ cutoff wavelength. Figure 8 shows an Arrhenius plot of the dynamic impedance-area product $R_{0} A$. The $R_{0} A$ value at $77 \mathrm{~K}$ was $824 \Omega \mathrm{cm}^{2}$.

\subsection{Responsivity measurement}

The responsivity was measured with a monochromator using a diffraction grating. The experimental setup is shown in Fig. 9. To estimate the absolute power of the measurement system, we used a thermopile detector (Dexter Model 2M) as a reference detector. Figure 10 shows the spectral responsivity of the T2SL single-pixel detector measured at $30 \mathrm{~K}$ and a $-20 \mathrm{mV}$ bias. The responsivity $R$ was approximately $0.33 \pm$ $0.05 \mathrm{~A} / \mathrm{W}$ at $4.5 \mu \mathrm{m}$. We also measured the responsivity at $70 \mathrm{~K}$. The responsivity at $4.5 \mu \mathrm{m}$ is the same within the error bars. The quantum efficiency was approximately $9 \%$. According to the T2SL modeling, the absorption coefficient $(=\alpha)$ of infrared light is approximately $2000 \mathrm{~cm}^{-1} \cdot{ }^{(10)}$ Because the thickness $(=d)$ of the 100-period T2SL layer was approximately $0.5 \mu \mathrm{m}$, the expected quantum efficiency was $11 \%$, which is almost the same as the measured quantum efficiency.

In comparison with a quantum well infrared photodetector (QWIP) or PtSi Schottkybarrier infrared detectors, the quantum efficiency of the T2SL is higher even though the thickness of T2SL layer is only $0.5 \mu \mathrm{m}$. This is an advantage of the T2SL infrared detector. This also implies that the quantum efficiency can be increased with a thicker T2SL layer because the quantum efficiency of T2SL is simply proportional to $1-\mathrm{e}^{-\alpha d}$. If we increase the thickness of T2SL to up to about $3.5 \mu \mathrm{m}$, the quantum efficiency would be over $50 \%$.

The cutoff wavelength (defined as the point where the responsivity dropped to $50 \%$ of the $4.5 \mu \mathrm{m}$ value) was $5.5 \mu \mathrm{m}$. This was near the designed $6 \mu \mathrm{m}$ cutoff wavelength.

From the dynamic impedance-area product and the spectral responsivity, we can obtain the Johnson noise limited detectivity $D^{*}$ as

$$
D^{*}=R \sqrt{\frac{R_{0} A}{4 k T}},
$$



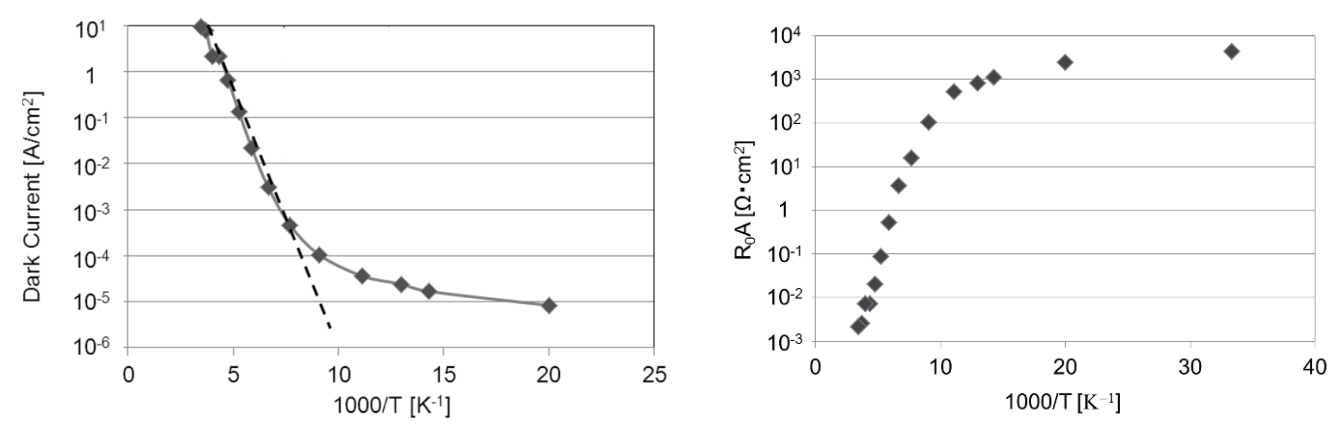

Fig. 7 (left). Arrhenius plot of dark current density (dark current density as a function of 1000/T). Fig. 8 (right). Arrhenius plot of dynamic impedance-area product $\left(R_{0} A\right.$ as a function of 1000/T).

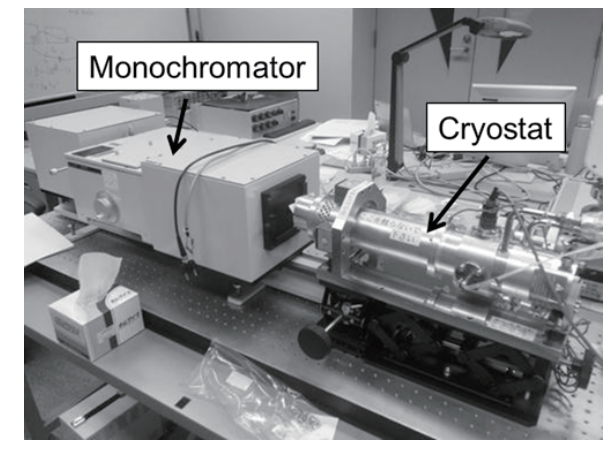

Fig. 9. Experimental setup for responsivity measurement.

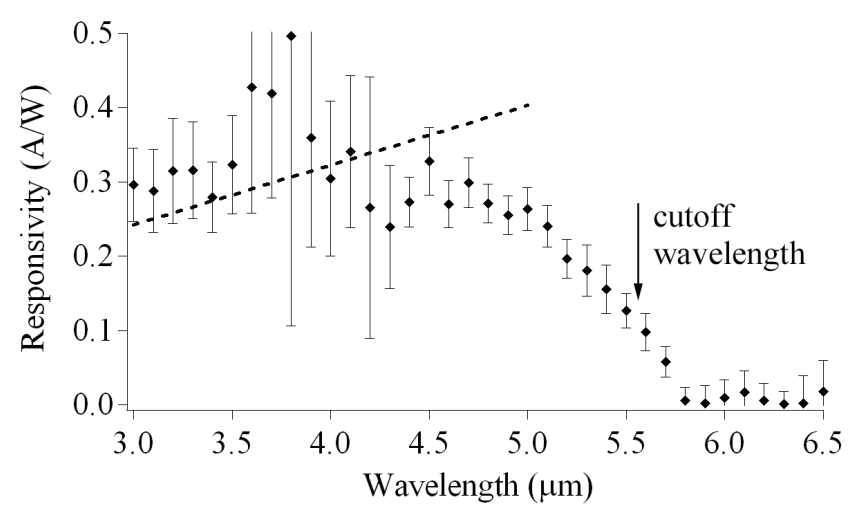

Fig. 10. Spectral responsivity of T2SL single-pixel detector. Dashed line indicates a quantum efficiency of $10 \%$. The arrow represents the cutoff wavelength. 
where $k$ is the Boltzmann constant and $T$ is the temperature. Using $R_{0} A$ derived in $\S$ 5.1, the detectivity $D^{*}$ is calculated as $1.4 \times 10^{11} \mathrm{~cm} \mathrm{~Hz}^{1 / 2} / \mathrm{W}$. Compared with a previous result of the T2SL MWIR detector, ${ }^{(9)}$ the detectivity of our detector is still two orders of magnitude lower than that of another T2SL MWIR detector $\left(D^{*}=1.5 \times 10^{13} \mathrm{~cm} \mathrm{~Hz}^{1 / 2} / \mathrm{W}\right.$ at $77 \mathrm{~K})$. We are planning to improve the performance of the detector. To increase the responsivity, we need to increase the thickness of the T2SL layers. The suppression of the dark current using a barrier structure is an important work for future VLWIR detector development.

\section{Fabrication of T2SL Array Detector}

We fabricated the T2SL array detector based on the single-pixel detector with the cutoff wavelength of $6 \mu \mathrm{m}$ described in $\S 4$. We increased the thickness of the T2SL to up to 300 periods to improve the responsivity. A commercial readout integrated circuit (ROIC), FLIR ISC0903, was selected. The pixel format and pixel size of ISC0903 are $320 \times 256$ and $30 \mu \mathrm{m}$, respectively. For a hybridization process, we used an indium solder bump and flip-chip bonding developed for a short-wave infrared T2SL detector. ${ }^{(11)}$ Following the hybridization with the ROIC, we lapped the detector mechanically to remove the GaSb substrate because the undoped GaSb substrate is not transparent in the MWIR region.

We used an F2.3 MWIR lens to evaluate the array detector. The array detector was cooled to $77 \mathrm{~K}$ using liquid nitrogen. A Pulse Instruments FPA test station was used for the driving and readout of the ROIC. Figure 11 shows an image of a face taken with the array detector. The background was subtracted, but a pixel-to-pixel nonuniformity correction was not performed. The integration time to acquire an image was $200 \mu \mathrm{s}$, and the frame rate was approximately $127 \mathrm{~Hz}$. Although we removed a GaSb substrate with

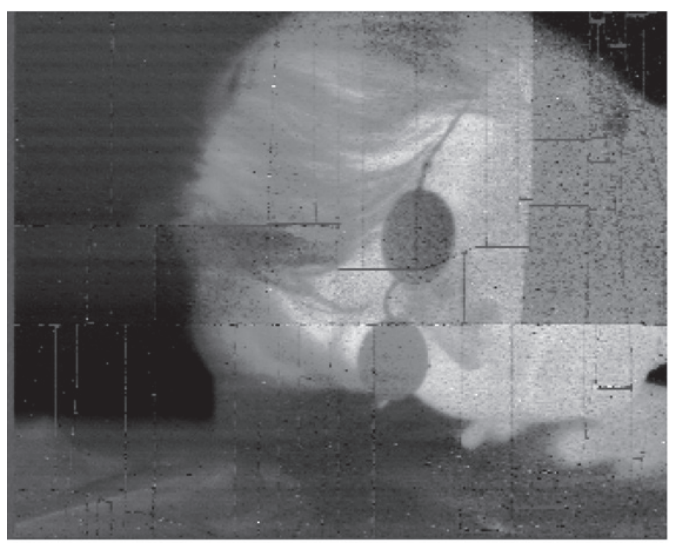

Fig. 11. Image of face obtained with $6 \mu \mathrm{m}$ array detector. 
a thickness of approximately $50 \mu \mathrm{m}$, as can be observed in the image, there are many cracks caused by the thermal mismatch between the T2SL detector and Si ROIC or an underfill material that is inserted between the detector and ROIC to remove the $\mathrm{GaSb}$ substrate. We used a flat blackbody to measure the noise-equivalent delta temperature $(N E d T)$. We derived the $N E d T$ using the 100-frame average and standard deviation of the image of 30 and $50{ }^{\circ} \mathrm{C}$ flat blackbody. Figure 12 shows the histogram of $N E d T$. The average of the $N E d T$ was $0.25 \mathrm{~K}$.

The relation between $N E d T$ and $D^{*}$ is given by

$$
D^{*}=\frac{4}{\pi} \frac{\sqrt{\Delta f} F^{2}}{\sqrt{A} \Delta \lambda \tau \frac{\partial B_{\lambda}(T)}{\partial T}} \frac{1}{N E d T},
$$

where $\Delta f$ is noise bandwidth, $F$ is the f-number of the optics, $A$ is the detector active area, $\Delta \lambda$ is the responsive waveband, $\tau$ is the transmittance of the optics, and $\partial B / \partial T$ is the temperature derivative of the Planck function. When we employ $\eta$ as $1.0, \lambda$ as $4 \mu \mathrm{m}, \Delta \lambda$ as $4 \mu \mathrm{m}(2-6 \mu \mathrm{m})$, and $\Delta f$ as $5 \mathrm{kHz}$ from the integration time, the expected $D^{*}$ derived from eq. (2) is $5.6 \times 10^{9} \mathrm{~cm} \mathrm{~Hz}^{1 / 2} / \mathrm{W}$, which is considerably lower than $D^{*}$ derived from the single-pixel detector described in $\S 5$. This implies that the performance of the array detector is not sufficient because of the higher dark current or lower responsivity in comparison with the single-pixel detector. The array detector fabrication process needs to be further improved to obtain a better performance. In particular, the $\mathrm{GaSb}$ substrate is still too thick to detect all photons because, as our measurement showed, the transmittance of the $50-\mu \mathrm{m}$-thick GaSb substrate is approximately $40 \%$ at $4.5 \mu \mathrm{m} .{ }^{(12)} \mathrm{We}$ are planning to remove all substrates by chemical etching in the next process.

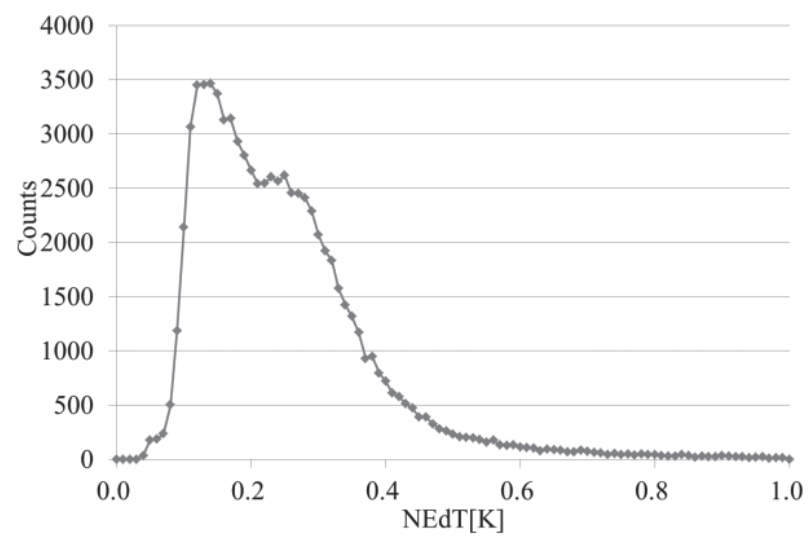

Fig. 12. Histogram of $N E d T$ of $6 \mu \mathrm{m}$ array detector. 


\section{Conclusion}

The current status of the T2SL detector development at JAXA is summarized in this paper. The fabricated MWIR pin diode detector shows that the dark current density was $1.8 \times 10^{-4} \mathrm{~A} / \mathrm{cm}^{2}$ at a bias voltage of $-100 \mathrm{mV}$, and the quantum efficiency was approximately $9 \%$ at a wavelength of $4.5 \mu \mathrm{m}$. However, compared with a previous work, our detector still does not show satisfactory performance. Suppression of the dark current using a barrier structure is important for future VLWIR detector development.

We also fabricated a T2SL array detector based on the single-pixel detector. The $D^{*}$ value derived from the array detector is considerably lower than that derived from the single-pixel detector, probably because this discrepancy is caused by the absorption of infrared light by the GaSb substrate. We are planning to remove all substrates using chemical etching in the next process.

Although we successfully demonstrated the MWIR array detector with a cutoff wavelength of $6 \mu \mathrm{m}$, further improvements are necessary by improving the epitaxial quality and process to realize array detectors with a cutoff wavelength of $15 \mu \mathrm{m}$.

\section{References}

1 Japan Aerospace Exploration Agency: EORC Annual Report 2008 No. 12, SP-09-017E (2008).

2 M. Field, G. J. Sullivan, A. Ikhlassi, C. Grein, M. E. Flatté, H. Yang, M. Zhong, and M. Weimer: Proc. SPIE 6127 (2006) 61270V.

3 D. L. Smith and C. Mailhiot: J. Appl. Phys. 62 (1987) 2545.

4 R. Rehm, M. Walther, J. Schmitz, J. Fleissner and F. Fuchs: Proc. SPIE 5783 (2005) 123.

5 M. Razeghi, E. K. Huang, B.-M. Nguyen, S. Ramezani-Darvish, S. A. Pour, G. Chen, A. Haddadi and M.-A. Hoang: Proc. SPIE 8012 (2011) 80120T.

6 A. Haddadi, S. R. Darvish, G. Chen, A. M. Hoang, B. M. Nguyen and M. Razeghi: AIP Conf. Proc. 1416 (2011) 56.

7 M. Walther, R. Rehm, J. Fleissner, J. Schmitz, J. Ziegler, W. Cabanski and R. Breiter: Proc. SPIE 6542 (2007) 654206.

8 R. Chaghi, C. Cervera, H. Ait-Kaci, P. Grech, J. B. Rodriguez and P. Christol: Semicond. Sci. Technol. 24 (2009) 065010.

9 Y. Wei, A. Hood, H. Yau, A. Gin, M. Razeghi, M. Z. Tidrow and V. Nathan: Appl. Phys. Lett. 86 (2005) 233106.

10 J. T. Olesberg, S. A. Anson, S. W. McCahon, M. E. Flatté, D. H. Chow and T. C. Hasenberg: Appl. Phys. Lett. 72 (1998) 229.

11 H. Inada, H. Mori, Y. Nagai, Y. Iguchi, T. Saitoh, K. Fujii, T. Ishizuka and K. Akita: Proc. SPIE 8012 (2011) 801220.

12 T. Takekawa: Master's thesis, Ritsumeikan University (2014) (in Japanese). 